\title{
Les fistules péniennes urétrocutanées postcirconcision : à propos de 85 cas
}

\section{Penile urethrocutaneous fistula postcircumcision: a series of 85 cases}

\author{
Y. Sow $\cdot$ B. Diao $\cdot$ Y.O. Tfeil $\cdot$ P.A. Fall $\cdot$ B. Fall \\ A.F. Kabore $\cdot$ A.K.H. Ndoye $\cdot$ B.A. Diagne
}

Reçu le 19 janvier 2009 ; accepté le 21 mars 2009

(C) Springer-Verlag 2009

Résumé Buts : Analyser les aspects cliniques et thérapeutiques des cas de fistules péniennes liés à une circoncision et pris en charge dans notre pratique quotidienne urologique. Patients et méthodes : Étude rétrospective des dossiers des patients admis pour fistule pénienne postcirconcision de la période allant de 1990 à 2007.

Résultats : Quatre-vingt-cinq cas ont été notés avec une moyenne d'âge de 10,7 ans (15 mois et 38 ans). La circoncision était réalisée par un personnel paramédical dans la majorité des cas. Le délai de consultation était en moyenne de 4,1 $\pm 5,3$ ans (1 semaine-28 ans). La fistule était associée à une amputation partielle du gland dans un cas et une sténose du méat urétral dans trois cas. Le traitement chirurgical a consisté surtout à un dédoublement urétrocutané. Avec un recul moyen de 3,35 ans (2 mois8 ans), nous avions noté $81,2 \%$ de bons résultats.

Conclusion: La fistule pénienne postcirconcision est fréquente dans nos régions. Sa prise en charge est assez codifiée, mais le risque d'échec n'est pas négligeable, d'où l'intérêt de la prévention.

\section{Mots clés Fistule $\cdot$ Pénis · Circoncision}

\begin{abstract}
Purpose: To assess clinical and therapeutic aspects of penile urethral fistula following circumcision in our daily urological practice.

Patients and methods: We carried out a retrospective study of cases of penile fistula between 1990 and 2007.

Results: A series of 85 cases is reported. The mean age was 10.7 years (15 months -38 months). The circumcision was performed by paramedical persons in the majority of cases. The mean period elapsing before consultation was
\end{abstract}

Y. Sow $(\bowtie) \cdot$ B. Diao - Y.O. Tfeil · P.A. Fall · B. Fall ·

A.F. Kabore - A.K.H. Ndoye - B.A. Diagne

Service d'urologie-andrologie, CHU Aristide-Le-Dantec,

BP 3001 Dakar, Sénégal

e-mail : yahyasowdj@yahoo.fr
$4.1 \pm 5.3$ years (range: 1 week-28 years). The fistula was associated with partial amputation of the glans penis in one case, and with urethral meatus stenosis in three cases. The surgical treatment was layered repair in the majority of cases. The average follow-up was 3.35 years (range: 2 months -8 years). Overall results were satisfactory in $81.2 \%$ of cases.

Conclusions: Penile fistula due to circumcision is common in our region. Its management is codified but there is a significant risk of failure, making prevention even more important.

Keywords Fistula $\cdot$ Penis $\cdot$ Circumcision

\section{Introduction}

La circoncision est une pratique ancienne dont l'origine exacte est inconnue et se perd dans la nuit des temps. Elle est largement pratiquée de par le monde avec des buts et des significations cependant différents [1]. La pratique répandue de la circoncision explique le fait qu'elle soit effectuée par des opérateurs variés, qualifiés ou non, surtout en Afrique. Cela, ajouté à la diversité des méthodes chirurgicales utilisées que ce soit en milieu médical ou non médical, rend compte de la possibilité de complications. Ces complications sont nombreuses et certaines sont gravissimes pouvant engager le pronostic fonctionnel sexuel et urinaire, voire le pronostic vital. Parmi celles-ci, nous pouvons citer :

- les hémorragies ;

- l'amputation du gland ;

- la sténose du méat urétral ;

- la fistule pénienne, etc.

Le but de notre étude était d'analyser les aspects cliniques et thérapeutiques des cas de fistules péniennes urétrocutanées secondaires à une circoncision et pris en charge dans notre pratique quotidienne urologique. 


\section{Patients et méthodes}

À partir d'une étude descriptive rétrospective, nous avons répertorié tous les cas de fistules péniennes urétrocutanées secondaires à une circoncision et reçus dans notre service, de la période allant de 1990 à 2007. Ainsi, pour chaque patient, les paramètres suivants ont été décrits :

- l'âge des patients ;

- le type de circoncision (circoncision médicale ou traditionnelle) ;

- le délai de consultation ;

- les motifs de consultation ;

- l'aspect clinique et les suites thérapeutiques avec comme critères d'appréciation des résultats :

- la qualité de la miction (présence de dysurie ou non, persistance ou non des fuites urinaires lors de la miction, etc.) ;

- l'aspect cosmétique du pénis.

\section{Résultats}

Durant cette période, 85 cas de fistules péniennes ont été notés. L'âge moyen des patients était de 10,7 $\pm 7,2$ ans avec des extrêmes de 15 mois à 38 ans. La circoncision était réalisée par un personnel paramédical dans 47 cas $(55,3 \%$ des cas) et par un tradipraticien dans 37 cas (43,5\% des cas), et dans un cas, il s'agissait d'une tentative de circoncision par une personne qui n'avait aucun statut qui lui permettait d'effectuer cette intervention.

Le délai de consultation était en moyenne de 4,1 $\pm 5,3$ ans avec des extrêmes d'une semaine et de 28 ans. Le motif de consultation était la fuite d'urines à travers un autre orifice lors de la miction. Dans trois cas, une dysurie était associée, et chez un patient, la consultation a été motivée par la survenue d'un dysfonctionnement érectile, et le diagnostic de fistule était posé à l'examen physique. La fistule était isolée dans la majorité des cas (81 cas) et siégeait toujours au niveau du sillon balanopénien (Fig. 1). Elle était associée à une amputation partielle du gland dans un cas et une sténose du méat urétral dans trois cas.

Le traitement de la fistule pénienne est chirurgical. Tous nos patients ont bénéficié d'une intervention chirurgicale. Cette dernière était réalisée sous anesthésie générale dans $71,7 \%$ des cas, sous anesthésie locale (bloc pénien) dans $15,3 \%$ des cas et sous anesthésie locorégionale (rachianesthésie ou anesthésie caudale) dans $13 \%$ des cas. Le mode de drainage après traitement chirurgical dépendait de l'opérateur et de la taille de la fistule. Ainsi, un drainage par sonde transurétrale a été effectué chez 42 patients $(49,4 \%$ de l'ensemble des fistules), un cathétérisme sus-pubien chez cinq patients $(5,9 \%$ des cas), alors que chez 38 patients

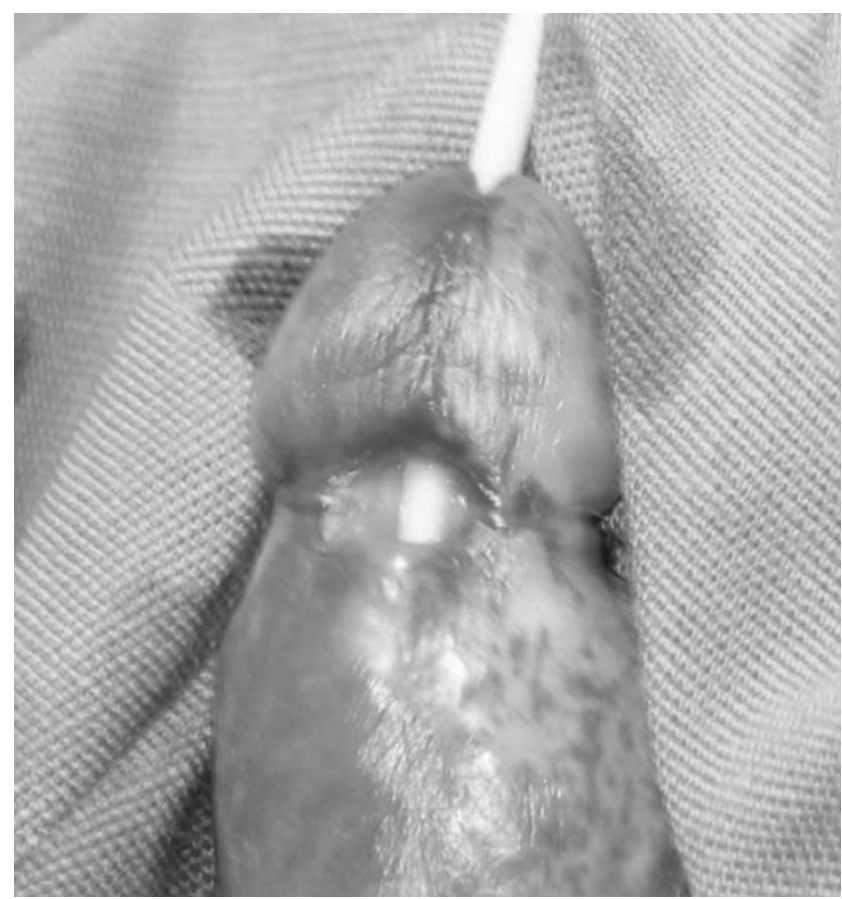

Fig. 1 Patient présentant une fistule urétrocutanée siégeant au niveau du sillon balanopénien (Diapothèque urologie HALD)

(44,7\% des cas), aucun drainage n'a été effectué. La fistule a été traitée par dédoublement urétrocutané chez 68 patients (80\% des cas), par confection d'un lambeau cutané de retournement chez 11 patients $(13 \%)$ et par urétrorraphie selon Davis chez six patients. Les trois cas associés de sténose du méat urétral ont bénéficié en plus d'une méatoplastie.

Les résultats thérapeutiques ont été appréciés selon les critères suivants :

- absence de fuite d'urines ;

- miction facile sans dysurie ;

- aspect cosmétique satisfaisant du pénis.

Avec un recul moyen de 3,35 ans et des extrêmes de deux mois et huit ans, les résultats globaux étaient satisfaisants dans $81,2 \%$ des cas. Le taux de complication était de $18,8 \%$, soit 16 cas. La complication la plus fréquemment notée était la fistule résiduelle (14 cas). Les différents types de complications postopératoires ainsi que les traitements complémentaires et le devenir des patients sont rapportés sur le Tableau 1.

\section{Discussion}

La fistule pénienne urétrocutanée est une complication bien connue de la circoncision. Au Sénégal, Ba et al. [2] et Sylla et al. [3] rapportaient déjà respectivement 20 et 41 cas. 
Tableau 1 Complications postopératoires, traitements complémentaires et suites

\begin{tabular}{lllll}
\hline Traitement initial & Complications & Nombre de cas & Traitement complémentaire & Suites \\
\hline Dédoublement urétrocutané & Lâchage de suture & 1 & Dédoublement urétrocutané & Simples \\
& Fistule résiduelle & 11 & Dédoublement urétrocutané & Simples \\
Lambeau cutané & Fistule résiduelle & 2 & Dédoublement urétrocutané & Simples \\
& Lâchage de suture & 1 & Lambeau cutané & Fistule résiduelle \\
Urétrorraphie de Davis & Fistule résiduelle & 1 & Dédoublement urétrocutané & Simples \\
Total & & 16 & & \\
\hline
\end{tabular}

Tableau 2 Fréquence de la fistule urétrale selon les auteurs

\begin{tabular}{lll}
\hline Auteurs (Pays) & Durée d'étude (ans) & Nombre de cas \\
\hline Baskin et al. [4] (États-Unis) & 7 & 8 \\
Diallo et al. [12] (Guinée) & 5 & 22 \\
Ceylan et al. [7] (Turquie) & 9 & 5 \\
Ba et al. [2] (Sénégal) & 2 & 13 \\
Sylla et al. [3] (Sénégal) & 11 & 41 \\
Notre série (Sénégal) & 17 & 85 \\
\hline
\end{tabular}

Elle est donc assez fréquente dans nos régions. Cependant, d'autres auteurs la qualifient de complication rare de la circoncision [4,5]. L'incidence de cette complication différemment rapportée par les auteurs est consignée sur le Tableau 2.

L'âge des patients est variable et dépend en général de l'âge à la circoncision et du délai écoulé entre la circoncision et la consultation. Dans notre série, l'âge moyen est élevé ; cela pouvant être lié non seulement à la période de la circoncision, qui est variable en fonction des us et coutumes (la circoncision traditionnelle est réalisée chez des adolescents, voire des adultes), mais aussi au délai de consultation qui est souvent long du fait de l'ignorance des possibilités thérapeutiques et du fatalisme. À l'inverse, aux États-Unis, Baskin et al. [4] retrouvent un âge moyen très bas (13 mois), car la circoncision est effectuée dans ces contrées à la période néonatale avec une consultation très précoce après complication.

La fistule siège presque toujours sur la face ventrale de la verge au niveau du sillon balanopénien $[2,3,5]$, car c'est à ce niveau que l'urètre, qui occupe la face antérieure du pénis, est le plus superficiel. Par contre, Agrawal et al. [6] rapportent des cas de fistules localisées au niveau de la face dorsale de la verge. Aussi, Ceylan et al. [7] notent un cas de fistule pénienne postcirconcision localisée au tiers moyen de la verge sans pouvoir cependant l'expliquer.

Des mécanismes variables expliquant la survenue de la fistule ont été avancés. Le mécanisme le plus courant est probablement la prise d'une partie du mur urétral par un point de suture trop profond au moment de l'hémostase de l'artère du frein $[6,8]$ avec comme conséquence une nécrose urétrale secondaire, puis une fistulisation. D'autres cependant pensent que la fistule serait favorisée par une infection locale [9] ou par l'existence d'une anomalie pénienne rare méconnue telle que le méga-urètre. Dans nos régions, la fistule pénienne est surtout favorisée par la circoncision traditionnelle [3], particulièrement celle qui procède à la taille de trois lambeaux préputiaux [10]. La fistule survient lors du temps antérieur. Elle peut être également due au manque de qualification ou à l'inexpérience du personnel paramédical qui effectue la circoncision, car le plus souvent méconnaissant les considérations anatomiques. Dans les pays développés, les techniques utilisant le procédé du Plastibell ou le clamp de Gomco sont plus pourvoyeuses de fistules urétrocutanées [5,10,11], cela est lié probablement à l'écrasement urétral par le dispositif et à l'ischémie urétrale qui en résulte.

La méthode de cure de la fistule la plus usitée dans notre série est la technique de dédoublement urétrocutané qui consiste, après séparation de l'urètre de la peau pénienne et excision des tissus sclérosés, en une suture en plans séparés de l'urètre puis de la peau. Cette même technique était aussi préférée par d'autres auteurs [7,12,13]. Baskin et al. [4], quant à eux, après fendage du gland permettant de transformer la fistule en une sorte d'hypospadias, procédaient en une réparation de la fistule en utilisant soit un lambeau cutané pénien de retournement type Mathieu, soit un lambeau cutané pédiculé de type Onlay-Island prélevé à la face ventrale ou dorsale du pénis, tandis que Ba et al. [2] préfèrent l'urétrorraphie de Davis qui consiste en une suture endo-urétrale de la fistule par du fil résorbable.

Le mode de drainage après cure de fistule est très discuté. Dans notre série, la majorité des patients $(n=42)$ avait bénéficié d'un drainage urinaire par une sonde transurétrale, alors que 38 patients n'avaient aucun drainage. Le mode de drainage est surtout guidé par la taille de la fistule. En effet, plus la fistule était grande, plus nous avions tendance à drainer. Certains auteurs [2,11] préfèrent le cathétérisme sus-pubien car, pour eux, celui-ci a l'avantage de laisser « à sec » la zone fraîchement réparée et éviterait les inconvénients d'un cathétérisme transurétral qui pourrait être source d'infections favorisant le lâchage des sutures. Diallo et al. [12], cependant, procédaient systématiquement à un drainage urinaire soit par cathétérisme sus-pubien, soit par sondage transurétral. Sen et al. [14], après comparaison 
entre le mode de drainage par sondage transurétral et le mode sans drainage, retrouvaient qu'il n'y avait pas de différence statiquement significative entre les deux choix en termes de résultats thérapeutiques ; cependant, l'absence de drainage offrait un délai d'hospitalisation court et évitait les infections locales. Aussi, nous pensons que ce drainage urinaire n'est nécessaire que dans les cas où la fistule est de grande taille ou lorsqu'il existe des difficultés opératoires.

Les résultats thérapeutiques étaient dans l'ensemble satisfaisants, avec $81,2 \%$ de réussite dans notre série. Ces résultats sont superposables à ceux obtenus par d'autres auteurs [2,13]. Baskin et al. [4] et Ceylan et al. [7] notaient dans leur série respective $100 \%$ de bons résultats.

La morbidité (18,8\% des cas) était surtout représentée par la fistule résiduelle qui a bénéficié d'un traitement complémentaire avec succès dans tous les cas, et par le lâchage des sutures. Ces mêmes complications sont rapportées par $\mathrm{Ba}$ et al. [2] et Diallo et al. [12].

\section{Conclusion}

La circoncision est l'intervention la plus couramment pratiquée de par le monde. Ces complications sont graves, car pouvant engager le pronostic fonctionnel urinaire et sexuel, voire le pronostic vital. La fistule pénienne est une complication fréquente de la circoncision dans nos régions. $\mathrm{Sa}$ prise en charge est assez codifiée, mais le risque d'échec n'est pas négligeable, surtout dans notre contexte. La prévention de ces complications est alors indispensable et consisterait à créer et à vulgariser des structures sanitaires, à favoriser une meilleure accessibilité à ces structures aux enfants à circoncire et enfin à médicaliser la circoncision dans nos régions.
Déclaration de conflit d'intérêt : Les auteurs déclarent ne pas avoir de conflit d'intérêt.

\section{Références}

1. Tilles G (1999) Histoire et géographie des circoncisions rituelles. Prog Urol 9:1148-1157

2. Ba M, Ndoye A, Fall A, et al (2000) Les fistules péniennes après circoncision : à propos de 20 observations. Andrologie 10:75-77

3. Sylla C, Diao B, Diallo AB, et al (2003) Les complications de la circoncision : à propos de 63 cas. Prog Urol 13:266-272

4. Baskin LS, Canning DA, Snyder HM 3rd, Duckett JW Jr (1997) Surgical repair of urethral circumcision injuries. J Urol 158:2269-2271

5. Williams N, Kapila L (1993) Complications of circumcision. Br J Surg 80:1231-1236

6. Agrawal A, Parelkar S, Shah H, et al (2008) Multiple circumferential urethrocutaneous fistulae: a rare complication of circumcision. J Pediatr Urol [Epub ahead of print]

7. Ceylan K, Burhan K, Yilmaz Y, et al (2007) Severe complications of circumcision: an analysis of 48 cases. J Pediatr Urol 3:32-35

8. Limaye RD, Hancock RA (1968) Penile urethral fistula, as a complication of circumcision. J Pediatr 72:105-106

9. Johnson S (1949) Persistent fistula following circumcision. US N Med Bull 49:120-122

10. Bitho MS, Sylla S, Toure K, et al (1975) Les accidents de la circoncision et de l'excision en milieu africain. Bull Soc Med Afr Noire 20:249-255

11. Williams N, Chell J, Kapila L (1993) Why are children referred for circumcision? BMJ 306:28

12. Diallo AB, Bah I, Barry M, et al (2006) La fistule pénienne après circoncision : à propos de 22 cas. Afr J Urol 12(3):152-155

13. El-Bahnasawy MS, El-Sherbiny MT (2002) Paediatric penile trauma. BJU Int 90:92-96

14. Sen B, Adayener C, Akyol I (2007) Repairing urethrocutaneous fistula in adults: is a catheter necessary? Urology 70(2): 239-241 